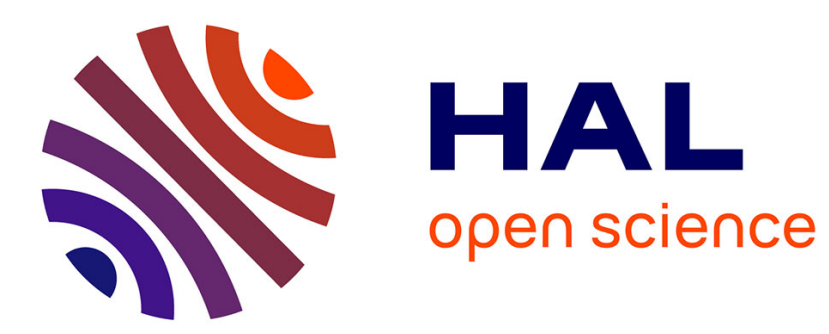

\title{
Optimal control of the orientation and alignment of an asymmetric-top molecule with terahertz and laser pulses \\ L. Coudert
}

\section{To cite this version:}

L. Coudert. Optimal control of the orientation and alignment of an asymmetric-top molecule with terahertz and laser pulses. Journal of Chemical Physics, 2018, 148 (9), pp.094306. 10.1063/1.5018914 . hal-02371709

\section{HAL Id: hal-02371709 \\ https://hal.science/hal-02371709}

Submitted on 14 Oct 2020

HAL is a multi-disciplinary open access archive for the deposit and dissemination of scientific research documents, whether they are published or not. The documents may come from teaching and research institutions in France or abroad, or from public or private research centers.
L'archive ouverte pluridisciplinaire HAL, est destinée au dépôt et à la diffusion de documents scientifiques de niveau recherche, publiés ou non, émanant des établissements d'enseignement et de recherche français ou étrangers, des laboratoires publics ou privés. 


\title{
Optimal control of the orientation and alignment of an asymmetric-top molecule with terahertz and laser pulses
}

\author{
L. H. Coudert* \\ Institut des Sciences Moléculaires d'Orsay (ISMO), CNRS, \\ Univ. Paris-Sud, Université Paris-Saclay, F-91405 Orsay, France
}

(Dated: October 14, 2020)

\begin{abstract}
Quantum optimal control theory is applied to determine numerically the terahertz and nonresonant laser pulses leading respectively to the highest degree of orientation and alignment of the asymmetric-top $\mathrm{H}_{2} \mathrm{~S}$ molecule. The optimized terahertz pulses retrieved for temperatures of zero and $50 \mathrm{~K}$ lead after $50 \mathrm{ps}$ to an orientation with $\left\langle\Phi_{Z x}\right\rangle=0.95973$ and $\left\langle\left\langle\Phi_{Z x}\right\rangle\right\rangle=0.74230$, respectively. For the zero temperature, the orientation is close to its maximum theoretical value; for the higher temperature it is below. The mechanism by which the terahertz pulse populates high lying rotational levels is elucidated. The $5 \mathrm{ps}$ long optimized laser pulse calculated for a zero temperature leads to an alignment with $\left\langle\Phi_{Z y}^{2}\right\rangle=0.94416$ and consists of several kick pulses with a duration of $\approx 0.1 \mathrm{ps}$. It is found that the timing of these kick pulses is such that it leads to an increase of the rotational energy of the molecule. The optimized laser pulse retrieved for a temperature of $20 \mathrm{~K}$ is $6 \mathrm{ps}$ long and yields a lower alignment with $\left\langle\left\langle\Phi_{Z y}^{2}\right\rangle\right\rangle=0.71720$.
\end{abstract}

\section{INTRODUCTION}

Controlling molecular rotational degrees of freedom is an important field of research with many promising applications. ${ }^{1,2}$ The most successful techniques developed so far employ intense nonresonant laser pulses and terahertz pulses. The former relies on the interaction between the electric field and the induced dipole ${ }^{3-5}$ and leads to molecular alignment; the latter is based on the coupling between the permanent dipole and the electric field $d^{6,7}$ and results in molecular orientation. With nonresonant laser pulses, a fairly large alignment with $\left\langle\cos ^{2} \theta\right\rangle=0.8$ could be reached for the iodine molecule. ${ }^{5}$ With terahertz pulses, the degree of orientation is usually smaller and a value of $\langle\cos \theta\rangle$ of the order of 0.005 was obtained at room temperature for the linear OCS and the symmetric-top methyle iodide molecules. ${ }^{7,8}$ Increased alignment or orientation can be obtained with various experimental techniques involving state-selected molecules, ${ }^{9}$ two-color laser fields, ${ }^{10}$ and combining an electrostatic field and laser pulses ${ }^{11-13}$ or terahertz and femtosecond laser pulses. ${ }^{14}$

Pulse shaping in the case of both laser ${ }^{15,16}$ and terahertz ${ }^{17}$ pulses in conjunction with quantum optimal control theory ${ }^{18-25}$ provides us with another mean to substantially enhance orientation or alignment. Such a procedure was shown, at least theoretically, to be very effective in the case of linear molecules. An alignment with $\left\langle\cos ^{2} \theta\right\rangle=0.99$ could be reached by Lapert et al. ${ }^{22}$ for the $\mathrm{CO}$ molecule and an orientation with $\langle\cos \theta\rangle=0.91$ could be attained by Salomon et $a l^{26}$ for the HCN molecule. Only a limited number of theoretical results are available for asymmetric-top molecules. An elliptically polarized nonresonant laser pulse was computed by Artamonov and Seideman ${ }^{27}$ to obtain three-dimensional alignment. The

* laurent.coudert@u-psud.fr alignment obtained is characterized by $\left\langle\cos ^{2} \chi\right\rangle,\left\langle\cos ^{2} \theta\right\rangle$, and $\left\langle\cos ^{2} \phi\right\rangle$ close to $0.76,0.55$, and 0.32 , respectively. Due to the fact that only eight parameters describing the pulse shape were optimized and that an assumed time dependence was taken for the electric field enveloppe, the alignment is smaller than that achieved by Lapert et $a l^{22}$ albeit for a linear molecule. More recently, with an optimized terahertz pulse designed at $0 \mathrm{~K}$ for the $\mathrm{H}_{2} \mathrm{~S}$ molecule ${ }^{28}$ an orientation with $\left\langle\Phi_{Z x}\right\rangle=0.93$ was attained without making any assumption on the time variation of the electric field. Compared to linear molecules, asymmetric-top molecules display no periodic revivals ${ }^{29}$ and are characterized by an additional degree of freedom described by the Euler angle $\chi$. There are no results about the maximum attainable field free orientation or alignment that can be achieved with an asymmetrictop molecule and the way the pulse interacts with the molecule is not known.

In this investigation, quantum optimal control is applied to find terahertz and nonresonant laser pulses leading respectively to the highest degree of orientation and alignment of an asymmetric-top molecule. The $\mathrm{H}_{2} \mathrm{~S}$ molecule is taken as a test case as it is spectroscopically very well characterized ${ }^{30}$ and displays a permanent dipole moment ${ }^{31}$ as well as large enough components of its polarizability tensor ${ }^{32}$ so that it can be used in both terahertz and nonresonant laser pulses experiments. In the first part of the paper, using the two-point boundaryvalue quantum control paradigm (TBQCP) of Ho and Rabitz $^{23}$ and its extended version for mixed states of Liao et al. ${ }^{25}$ the optimal control theory equations are solved to derive the terahertz pulse leading to the largest degree of orientation of an ensemble of molecules initially described by a temperature $\mathcal{T}$. In the second part of the paper, using the monotonically convergent algorithm of Lapert et al. ${ }^{21}$ designed for Stark coupling beyond the linear dipole interaction, the nonresonant laser pulse allowing us to achieve the highest alignment of an ensemble of molecules is calculated. In both parts, the maximum 
attainable field free orientation or alignment is evaluated and its temperature dependence is calculated. The mechanism by which the pulse interacts with the molecule is also investigated.

In Section II, the calculation of the rotational energy levels of the semi-rigid asymmetric-top $\mathrm{H}_{2} \mathrm{~S}$ molecule is presented and matrix elements arising in the second order Stark coupling Hamiltonian are derived. In Sections III and IV, the optimized terahertz and laser pulses are derived and the mechanism allowing high lying rotational to be populated is elucidated. Section $\mathrm{V}$ is the conclusion.

\section{THEORY}

In this paper, we only consider degrees of freedom corresponding to the overall rotation of an asymmetrictop molecule; vibrational degrees of freedom are ignored. These rotational degrees of freedom are parameterized by the three usual Eulerian angles describing the orientation of the molecule-fixed $x y z$ axis system with respect to the laboratory-fixed $X Y Z$ axis system. The rotational energy calculation and the matrix elements of the Stark coupling Hamiltonian are dealt with below.

\section{A. Rotational energy levels}

The $\mathrm{H}_{2} \mathrm{~S}$ asymmetric-top $C_{2 v}$ molecule is attached to the molecule-fixed axis system using the $\mathrm{I}^{r}$ representation. ${ }^{33}$ Its two-fold axis of symmetry and molecular plane are the $x$ axis and the $x z$ plane, respectively. Since in this light molecule centrifugal distortion effects are important, they are taken into account in the rotational energy calculation and incorporated in the effective rotational Hamiltonian. The latter is written with the $A$ reduced form proposed by Watson ${ }^{34-36}$ taking the following form:

$$
\begin{aligned}
H_{\mathrm{R}}= & B J_{x}^{2}+C J_{y}^{2}+A J_{z}^{2}-\Delta_{K} J_{z}^{4}-\Delta_{K J} \mathbf{J}^{2} J_{z}^{2} \\
& -\Delta_{J} \mathbf{J}^{4}-\left\{\delta_{K} J_{z}^{2}+\delta_{J} \mathbf{J}^{2}, J_{x y}^{2}\right\}+H_{K} J_{z}^{6} \\
& +H_{K K J} \mathbf{J}^{2} J_{z}^{4}+H_{K J J} \mathbf{J}^{4} J_{z}^{2}+H_{J} \mathbf{J}^{6} \\
& +\left\{h_{K} J_{z}^{4}+h_{K J} \mathbf{J}^{2} J_{z}^{2}+h_{J} \mathbf{J}^{4}, J_{x y}^{2}\right\}+\cdots,
\end{aligned}
$$

where $J_{x}, J_{y}$, and $J_{z}$ are dimensionless molecule-fixed components of the rotational angular momentum; $J_{x y}^{2}=$ $J_{x}^{2}-J_{y}^{2} ;\{$,$\} is the anticommutator; A, B$, and $C$ are the rotational constants; and $\Delta_{K}, \Delta_{K J}, \ldots, h_{J}$ are distortion constants. The matrix of the Hamiltonian in Eq. (1) can be setup using symmetric top rotational functions $|J, k, M\rangle$ defined in agreement with Wigner ${ }^{37}$ in Eq. (1.43) of Svidzinskii ${ }^{38}$ and in Eq. (8-67) of Bunker's book. ${ }^{33}$ These functions are eigenfunctions of the total rotational angular momentum $\mathbf{J}^{2}$, of its molecule-fixed component $J_{z}$, and of its laboratory-fixed component $J_{Z}$ with eigenvalues $J(J+1), k$, and $M$, respectively. The
Table I. Symmetry species of the $\left|K^{\alpha}, J, M\right\rangle$ rotational functions $^{\text {a }}$

\begin{tabular}{cccccccc}
\hline \hline & & & \multicolumn{2}{c}{$J$ even } & & \multicolumn{2}{c}{$J$ odd } \\
\cline { 5 - 7 } \cline { 6 - 8 } Label & $K^{\mathrm{b}}$ & $\alpha$ & $\Gamma\left(C_{2 v}\right)$ & $\Gamma\left(C_{2}\right)$ & & $\Gamma\left(C_{2 v}\right)$ & $\Gamma\left(C_{2}\right)$ \\
\hline$E^{+}$ & $e$ & +1 & $A_{1}$ & $A$ & & $B_{1}$ & $B$ \\
$E^{-}$ & $e$ & -1 & $B_{1}$ & $B$ & & $A_{1}$ & $A$ \\
$O^{+}$ & $o$ & +1 & $A_{2}$ & $A$ & & $B_{2}$ & $B$ \\
$O^{-}$ & $o$ & -1 & $B_{2}$ & $B$ & & $A_{2}$ & $A$ \\
\hline \hline
\end{tabular}

a Symmetry species in the $C_{2 v}$ and $C_{2}$ symmetry groups for the Wang-type rotational functions introduced in Section II A.

b The symbols $e$ and $o$ stand for even and odd, respectively.

matrix of the rotational Hamiltonian in Eq. (1) can be split into 4 submatrices using the $\left|K^{\alpha}, J, M\right\rangle$ Wang-type linear combinations ${ }^{39}$ of $|J, k, M\rangle$ functions in Eq. (20) of Coudert. ${ }^{40}$ As indicated by Table I, these linear combinations are labeled $E^{+}, E^{-}, O^{+}$, and $O^{-}$, depending on the parity of $K$ and the value of $\alpha$, and can be assigned a symmetry species of the $C_{2 v}$ and $C_{2}$ symmetry groups. ${ }^{33}$ Rotational functions belonging to the $A$ and $B$ symmetry species of the latter group have a statistical weight $^{28} g$ equal to 1 and 3 , respectively. Numerical diagonalization of the submatrices yields asymmetric-top rotational energy levels and eigenfunctions labeled using the rotational quantum number $J$, the pseudo rotational quantum numbers $K_{a}$ and $K_{c}$, and the quantum number $M$. The rotational energy $E_{\text {Rot }}\left(J K_{a} K_{c}\right)$ does not depend on the latter. The symmetry species in $C_{2 v}$ of the rotational levels depends on the parity of $K_{a}$ and $K_{c}$ and can be found in Table I of Coudert. ${ }^{28}$

\section{B. Stark coupling Hamiltonian}

The Stark coupling Hamiltonian $H_{\mathrm{S}}$ accounts for the linear coupling of the electric field with the dipole moment and for the quadratic coupling described by the polarizability tensor:

$$
H_{\mathrm{S}}=-\boldsymbol{\mu} \cdot \mathbf{E}-\frac{1}{2} \mathbf{E} \cdot \boldsymbol{\alpha} \cdot \mathbf{E},
$$

where $\mathbf{E}$ is the electric field; $\boldsymbol{\mu}$ the dipole moment; and $\boldsymbol{\alpha}$ is the $3 \times 3$ symmetrical polarizability tensor. Matrix elements of the linear coupling term in Eq. (2) between two asymmetric-top rotational wavefunctions can be found in Eqs. (4)-(7) of Coudert ${ }^{28}$ where they are expressed in terms of the laboratory-fixed components of the electric field and of the molecule-fixed components of the dipole moment. For the quadratic coupling term in Eq. (2), the matrix elements can be similarly retrieved using tensorial operator algebra. ${ }^{41}$ There arise two rank 2 tensorial operators $E^{(2)}$ and $\alpha^{(2)}$ and two rank 0 tensorial operators $E^{(0)}$ and $\alpha^{(0)}$. Their spherical components are expressed in terms of Cartesian coordinates in Table II. Defining the scalar product of two irreducible tensor operators as in Eq. (5.2.4) of Edmonds, ${ }^{41}$ the last term in Eq. (2) can 
be written as the sum of two such products:

$$
\mathbf{E} \cdot \boldsymbol{\alpha} \cdot \mathbf{E}=E^{(2)} \cdot \alpha^{(2)}+E^{(0)} \cdot \alpha^{(0)} .
$$

Evaluation of the matrix elements between two asymmetric-top eigenfunctions of the $E^{(2)} \cdot \alpha^{(2)}$ term in this equation leads to the following results when laboratory-fixed spherical components are used:

$$
\begin{aligned}
& \left\langle J K_{a} K_{c}, M\left|E^{(2)} \cdot \alpha^{(2)}\right| J^{\prime} K_{a}^{\prime} K_{c}^{\prime}, M^{\prime}\right\rangle=\sum_{q=-2}^{+2}(-1)^{q} \\
& \times E_{-q}^{(2)}\left\langle J K_{a} K_{c}, M\left|\alpha_{q}^{(2)}\right| J^{\prime} K_{a}^{\prime} K_{c}^{\prime}, M^{\prime}\right\rangle
\end{aligned}
$$

As the asymmetric-top eigenfunctions are expanded in terms of symmetric top rotational functions, evaluation of the last term of Eq. (4) leads to matrix elements of the form $\left\langle J, k, M\left|\alpha_{q}^{(2)}\right| J^{\prime}, k^{\prime}, M^{\prime}\right\rangle$. Using Eq. (5.4.1) of Edmonds, ${ }^{41}$ such matrix elements can be rewritten:

$$
\begin{aligned}
& \left\langle J, k, M\left|\alpha_{q}^{(2)}\right| J^{\prime}, k^{\prime}, M^{\prime}\right\rangle=(-1)^{J-M} \\
& \quad \times\left(\begin{array}{ccc}
J^{\prime} & J & 2 \\
M^{\prime} & -M & q
\end{array}\right)\left\langle J, k\left\|\alpha^{(2)}\right\| J^{\prime}, k^{\prime}\right\rangle .
\end{aligned}
$$

The reduced matrix element in this equation can be retrieved from Eqs. (3.24) of Svidzinskii: ${ }^{38}$

$$
\begin{gathered}
\left\langle J, k\left\|\alpha^{(2)}\right\| J^{\prime}, k^{\prime}\right\rangle=\left[(2 J+1)\left(2 J^{\prime}+1\right)\right]^{1 / 2} \\
\times(-1)^{J-k}\left(\begin{array}{ccc}
J^{\prime} & J & 2 \\
k^{\prime} & -k & p
\end{array}\right) \mathrm{MF}_{\alpha_{p}^{(2)}}
\end{gathered}
$$

where $k^{\prime}-k+p=0$ and $\mathrm{MF}_{\alpha_{p}}^{(2)}$ denotes the constant molecule-fixed spherical components of $\alpha^{(2)}$. They can be expressed in terms of Cartesian coordinates using Table II. Evaluation of the matrix elements between two asymmetric-top functions of the $E^{(0)} \cdot \alpha^{(0)}$ term in Eq. (3) is straightforward. As rank 0 tensorial operators are involved, diagonal matrix elements arise:

$$
\begin{gathered}
\left\langle J K_{a} K_{c}, M\left|E^{(0)} \cdot \alpha^{(0)}\right| J^{\prime} K_{a}^{\prime} K_{c}^{\prime}, M^{\prime}\right\rangle= \\
E_{0}^{(0)} \alpha_{0}^{(0)} \delta_{J, J^{\prime}} \delta_{M, M^{\prime}} \delta_{K_{a}, K_{a}^{\prime}} \delta_{K_{c}, K_{c}^{\prime}} \cdot
\end{gathered}
$$

In the remaining sections, the matrix of $H_{\mathrm{S}}$ will be setup using a maximum value of $J$ denoted $J_{\text {Max }}$. The number of field-free rotational levels arising for a given $M$ value and a given symmetry species $\Gamma$ depends on $J_{\text {Max }}$ and $|M|$; it will be denoted $p(\Gamma,|M|)$. The optimized terahertz and laser pulses calculated below depend on $J_{\text {Max }}$. In each calculation, the value adopted for $J_{\text {Max }}$ ensures converged results, that is, increasing $J_{\text {Max }}$ does not alter the final orientation or alignment by more than $0.1 \%$.

The spectroscopic constants used to compute rotational energies with the effective Hamiltonian in Eq. (1) are given in a table available as supplementary material. The value taken for the permanent dipole moment component along the molecule-fixed $x$ axis $\mu_{x}$ is
Table II. Spherical components ${ }^{\mathrm{a}}$ of the tensorial operators in Eqs. (3)-(7)

\begin{tabular}{c|cc}
\hline \hline$q$ & $E_{q}^{(2)}$ & \multicolumn{1}{c}{$\alpha_{q}^{(2)}$} \\
\hline \pm 2 & $\left(E_{X}^{2}-E_{Y}^{2}\right) / 2 \pm i E_{X} E_{Y}$ & $\left(\alpha_{X X}-\alpha_{Y Y}\right) / 2 \pm i \alpha_{X Y}$ \\
\pm 1 & $\mp E_{X} E_{Z}-i E_{Y} E_{Z}$ & $\mp \alpha_{X Z}-i \alpha_{Y Z}$ \\
0 & $\left(2 E_{Z}^{2}-E_{X}^{2}-E_{Y}^{2}\right) / \sqrt{6}$ & $\left(2 \alpha_{Z Z}-\alpha_{X X}-\alpha_{Y Y}\right) / \sqrt{6}$ \\
\hline & $E_{q}^{(0)}$ & $\alpha_{q}^{(0)}$ \\
\hline 0 & $-\left(E_{X}^{2}+E_{Y}^{2}+E_{Z}^{2}\right) / \sqrt{3}$ & $-\left(\alpha_{X X}+\alpha_{Y Y}+\alpha_{Z Z}\right) / \sqrt{3}$ \\
\hline \hline
\end{tabular}

a Laboratory-fixed spherical components are given in terms of Cartesian coordinates. For molecule-fixed spherical components, the subscripted upper case $X, Y$, and $Z$ should be replaced by lower case letters.

0.978 Debye. $^{31}$ A strong electric field will lead to orientation of this axis in the direction of the electric field. ${ }^{28}$ For the three nonvanishing components of the polarizability tensor, $\alpha_{x x}, \alpha_{y y}$, and $\alpha_{z z}$, the values taken $\operatorname{are}^{32}$ $3.841,3.955$, and $3.863 \AA^{3}$, respectively. As $\alpha_{y y}$ is the largest diagonal component, a strong linearly polarized laser field will lead to an alignment of the molecule-fixed $y$ axis parallel to the axis of polarization. ${ }^{29}$

\section{OPTIMIZED TERAHERTZ PULSE}

The electric field of the terahertz pulse, taken parallel to the laboratory-fixed $Z$ axis, is written $\mathcal{E}(t)=E(t) \mathbf{i}_{Z}$, where $E(t)$ is the amplitude of the field and $\mathbf{i}_{Z}$ is the unit vector along the laboratory-fixed $Z$ axis. The electric field is optimized so as to maximize the orientation of the molecule-fixed $x$ axis along the laboratory-fixed $Z$ axis. Retaining only the linear coupling term in the Stark coupling Hamiltonian of Eq. (2), the time-dependent Hamiltonian describing the molecule is written:

$$
H(t)=H_{\mathrm{R}}-\mu_{x} E(t) \Phi_{Z x},
$$

where $\Phi_{Z x}=-\cos \chi \sin \theta$ is a direction cosine. The Hamiltonian in Eq. (8) belongs to the completely symmetrical $A$ symmetry species of $C_{2}$ and only displays $\Delta M=0$ matrix elements. Its matrix with the $|J, k, M\rangle$ basis set functions of Section II A can be split into $2 J_{\text {Max }}+1$ submatrices identified by $M$. Each submatrix is of $n \times n$ dimensions, with $n=\left(J_{\mathrm{Max}}+1\right)^{2}-M^{2}$, and can be split into two blocks corresponding to the $A$ and $B$ symmetry species of $C_{2}$. Each block is of $p \times p$ dimensions where $p$ is $p(A,|M|)$ or $p(B,|M|)$. For $M=0$, both blocks can be further split into two sub-blocks because the Stark coupling operator in the Hamiltonian of Eq. (8) then only displays nonvanishing $|\Delta J|=1$ matrix elements. The resulting four sub-blocks are labeled $A_{e}$, $A_{o}, B_{e}$, and $B_{o}$ and the relevant Wang-type functions 
Table III. $\left|K^{\alpha}, J, M\right\rangle$ rotational functions ${ }^{\text {a }}$ for $M=0$

\begin{tabular}{ccc|ccc}
\hline \hline Label $^{\mathrm{b}}$ & $J$ even & $J$ odd & Label $^{\mathrm{b}}$ & $J$ even & $J$ odd \\
\hline$A_{e}$ & $E^{+}$ & $O^{-}$ & $B_{e}$ & $E^{-}$ & $O^{+}$ \\
$A_{o}$ & $O^{+}$ & $E^{-}$ & $B_{o}$ & $O^{-}$ & $E^{+}$ \\
\hline \hline
\end{tabular}

a The Wang-type $\left|K^{\alpha}, J, M\right\rangle$ functions allowing us to block diagonalize the Hamiltonian in Eq. (8) into four sub-blocks for $M=0$.

b The label of each sub-block is given in this column.

appear in Table III. The same block diagonalization is valid for other operators and is used below for the direction cosine $\Phi_{Z x}$, the density matrix $\rho(t)$, and the time evolution operator $U\left(t, t^{\prime}\right)$.

\section{A. Monotonically convergent algorithm}

Applying the TBQCP of Ho and Rabitz ${ }^{23}$ requires choosing the operator $O(t)$ introduced in their Section II. Using their Eq. (A9) and accounting for the symmetry properties of the Hamiltonian in Eq. (8), we express $O(t)$ as:

$$
O(t)=\sum_{\Gamma=A, B} \sum_{M=-J_{\mathrm{Max}}}^{+J_{\mathrm{Max}}} \sum_{k} \sigma_{k}^{M, \Gamma}\left|\chi_{k}^{M, \Gamma}(t)\right\rangle\left\langle\chi_{k}^{M, \Gamma}(t)\right|,
$$

where $\sigma_{k}^{M, \Gamma}$ are positive constants and $\left|\chi_{k}^{M, \Gamma}(t)\right\rangle$ are orthogonal wavefunctions characterized by $M$, a $C_{2}$ symmetry species $\Gamma$, and the index $k$, which are solutions of the time-dependent Schrödinger equation with the Hamiltonian in Eq. (8). Equation (9) ensures that the operator $O(t)$ is positive semidefinite, hermitian, and satisfies the invariant equation. ${ }^{42}$

The extended TBQCP for mixed states of Liao et al. ${ }^{25}$ leads to a monotonically convergent algorithm based on their Eq. (21). This equation, rewritten below, allows us to express the optimized field $E(t)$ in term of the initial field $E^{(0)}(t)$ according to:

$$
E(t)=E^{(0)}(t)+\eta S(t) f_{\rho}(t)
$$

where $\eta$ is a positive constant; $S(t)$ is the field envelope; and $f_{\rho}(t)$ is a functional of the electric field. In agreement with Eq. (19) of Liao et al. ${ }^{25}$ it should be expressed in terms of the operator $O(t)$ in Eq. (9) and of the density matrix $\rho(t)$ as:

$$
f_{\rho}(t)=-\frac{1}{i \hbar} \operatorname{Tr}\left\{\left[O(t), \mu_{x} \Phi_{Z x}\right] \rho(t)\right\},
$$

where [,] is the commutator. The density matrix satisfies the von Neumann equation for the Hamiltonian in Eq. (8). At time $T$, the operator $O(t)$ in Eq. (9) should coincide $^{23}$ with a specific physical operator $O_{T}$ so as to maximize the thermal average $\left\langle\left\langle O_{T}\right\rangle\right\rangle$. In the present case, maximum orientation is sought and $O_{T}$ is taken equal to

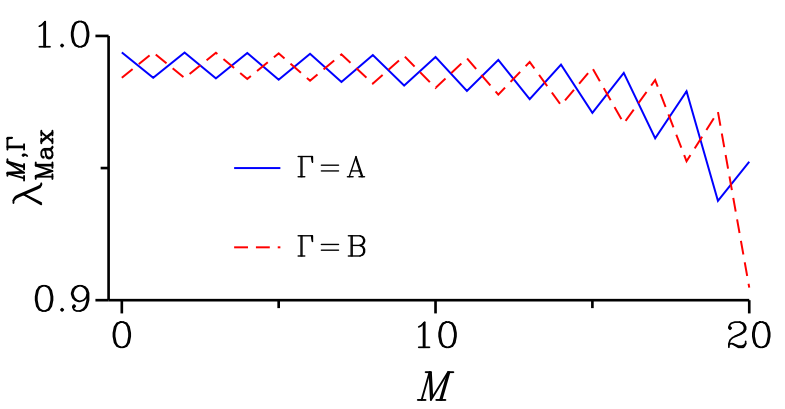

Figure 1. The largest eigenvalue $\lambda_{\mathrm{Max}}^{M, \Gamma}$ of the direction cosine $\Phi_{Z x}$ as a function of $M$ for $J_{\mathrm{Max}}=20$. A solid (dashed) line corresponds to the $C_{2}$ symmetry species $A(B)$.

$\mathbf{1}+\Phi_{Z x}$, where $\mathbf{1}$ is the unity operator. The operator $O_{T}$ can be expressed in terms of the eigenfunctions $\left|\phi_{k}^{M, \Gamma}\right\rangle$ and eigenvalues $\lambda_{k}^{M, \Gamma}$ of $\Phi_{Z x}$ as:

$$
O_{T}=\sum_{\Gamma=A, B} \sum_{M=-J_{\operatorname{Max}}}^{+J_{\operatorname{Max}}} \sum_{k=1}^{p(\Gamma,|M|)}\left(1+\lambda_{k}^{M, \Gamma}\right)\left|\phi_{k}^{M, \Gamma}\right\rangle\left\langle\phi_{k}^{M, \Gamma}\right| .
$$

Comparing this equation with Eq. (9) shows that $\left|\chi_{k}^{M, \Gamma}(T)\right\rangle=\left|\phi_{k}^{M, \Gamma}\right\rangle$ and that $\sigma_{k}^{M, \Gamma}=1+\lambda_{k}^{M, \Gamma}$, ensuring $\sigma_{k}^{M, \Gamma}>0$.

Eigenvalues and eigenfunctions of $\Phi_{Z x}$ should be computed to obtain the operator $O(t)$. The matrix of $\Phi_{Z x}$ is set up with the $|J, k, M\rangle$ basis set functions of Section II A, using the matrix elements in Eqs. (4)-(7) of Coudert. ${ }^{28}$ This matrix can be block-diagonalized in the same way as the Hamiltonian in Eq. (8). Figure 1 shows $\lambda_{\text {Max }}^{M, \Gamma}$ the largest eigenvalues of $\Phi_{Z x}$ for $J_{\text {Max }}=20$, $0 \leq M \leq J_{\mathrm{Max}}$, and both $C_{2}$ symmetry species. A fast variation with the parity of $M$ superimposed on a slower decrease with $M$ can be seen. For an even (odd) value of $M$, the largest eigenvalue for $\Gamma=A$ is larger (smaller) than that for $\Gamma=B$. Figure 1 emphasizes that the largest eigenvalue of $\Phi_{Z x}$ is obtained for $M=0$ and $\Gamma=A$; its eigenfunction involves $A_{e}$-type rotational functions.

The maximum orientation for a given temperature $\mathcal{T}$ can be estimated, as Liao et al. ${ }^{25}$ expressing $\rho(T)$ the density matrix at time $T$ with the eigenfunctions of $\Phi_{Z x}$ :

$$
\rho_{\mathrm{opt}}=\sum_{\Gamma=A, B} \sum_{M=-J_{\mathrm{Max}}}^{+J_{\mathrm{Max}}} \sum_{k=1}^{p(\Gamma,|M|)} \omega_{n(k)}^{\Gamma, J(k)}\left|\phi_{k}^{M, \Gamma}\right\rangle\left\langle\phi_{k}^{M, \Gamma}\right|,
$$

where $\omega_{n}^{\Gamma, J}$ is the Boltzmann factor for the field-free rotational level characterized by a $C_{2}$ symmetry species $\Gamma$, a rotational quantum number $J \geq|M|$, and the index $n$. For each symmetry species $\Gamma$ and for each $M$ value, there arises $p(\Gamma,|M|)$ field-free rotational levels and eigenvalues $\lambda_{k}^{M, \Gamma}$. The one to one correspondence between $J, n$ and $k$ allows us to choose the functions $J(k)$ and $n(k)$ in Eq. (13) so that the lowest lying field-free levels are associated with the largest eigenvalues. This choice leads 


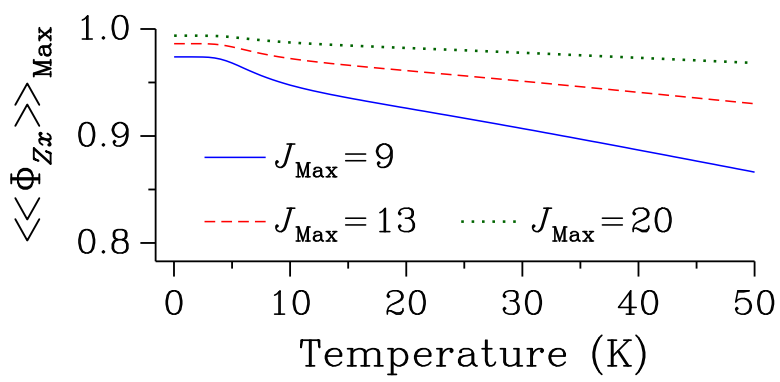

Figure 2. The largest orientation $\left\langle\left\langle\Phi_{Z x}\right\rangle\right\rangle_{\operatorname{Max}}$, defined in Eq. (14), is plotted as a function of the temperature in Kelvin for $J_{\mathrm{Max}}=9,13$, and 20, in solid, dashed, and dotted lines, respectively.

to the largest value of the thermal average of $\Phi_{Z x}$ :

$$
\left\langle\left\langle\Phi_{Z x}\right\rangle\right\rangle_{\operatorname{Max}}=\sum_{\Gamma=A, B} \sum_{M=-J_{\operatorname{Max}}}^{+J_{\operatorname{Max}}} \sum_{k=1}^{p(\Gamma,|M|)} \omega_{n(k)}^{\Gamma, J(k)} \lambda_{k}^{M, \Gamma} .
$$

Figure 2 shows the variations of $\left\langle\left\langle\Phi_{Z x}\right\rangle\right\rangle_{\text {Max }}$ with the temperature for several value of $J_{\mathrm{Max}}$. For a given temperature, it can be seen that taking a larger $J_{\text {Max }}$ leads to a larger maximum orientation. For a given value of $J_{\mathrm{Max}}$, the maximum orientation decreases with the temperature.

\section{B. Numerical results}

In the iterative procedure, ${ }^{23}$ the optimization time $T$ was set to $50 \mathrm{ps}$ and 1024 time intervals of equal duration were used for the computation of the time evolution of the density matrix and of $O(t)$ in Eq. (9). The field correction was computed using Eq. (49) of Ho and Rabitz ${ }^{23}$ where $f_{\mu}^{(n+1)}(t)$ was obtained from Eq. (11) of the present paper, the parameter $\eta$ was set to 1 , and the field envelope $S(t)$ was taken as:

$$
S(t)=\frac{A}{1+500[(2 t-T) / T]^{16}} .
$$

The constant $A$ is 1500 for the zero temperature and 2100 for the temperature of $50 \mathrm{~K}$, expressing the electric field in $\mathrm{kV} / \mathrm{cm}$. The electric field was optimized starting from a zero initial field $E^{(0)}(t)$.

\section{Zero temperature}

For a zero temperature, only one wavefunction arises and the density matrix reduces to $\rho(t)=|\psi(t)\rangle\langle\psi(t)|$. For $t=0,|\psi(t)\rangle$ is the $A_{e}$-type wavefunction of the $0_{00}, M=0$ ground rotational level. For $t>0,|\psi(t)\rangle$ remains an $A_{e}$-type wavefunction due to the block decomposition of the time evolution operator. Taking $J_{\mathrm{Max}}=9$, convergence was reached after 600 iterations and the average value of $\Phi_{Z x}$ at the end of the pulse was 0.95973 .
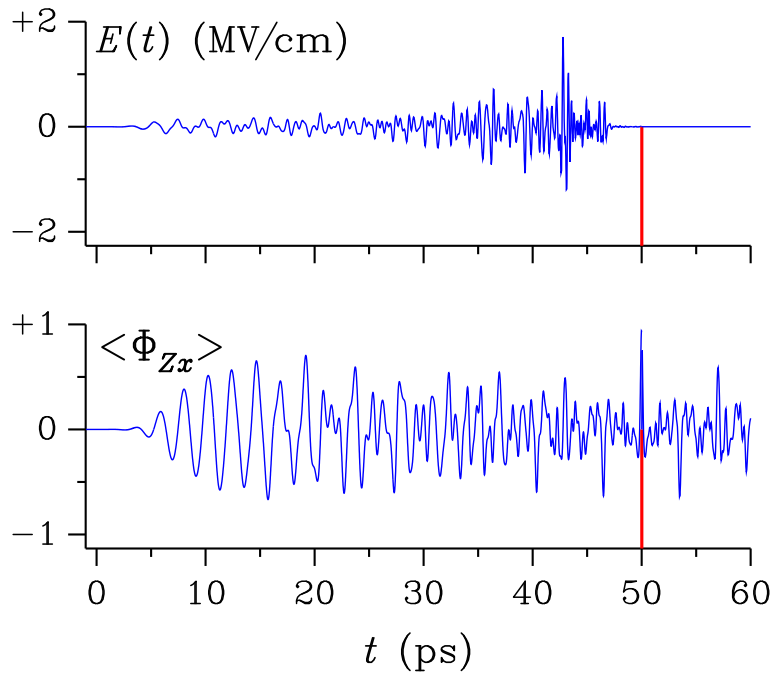

Figure 3. The optimized electric field $E(t)$ obtained for a temperature of $0 \mathrm{~K}$ in Section III B 1 (top panel) and the average value $\left\langle\Phi_{Z x}\right\rangle$ (lower panel) as a function of the time $t$ in ps. For the optimization time $T=50 \mathrm{ps}$, indicated by a vertical line in both panels, $\left\langle\Phi_{Z x}\right\rangle=0.95973$.

This number should be compared to 0.97391 , the value of $\lambda_{\text {Max }}^{M, \Gamma}$ for $M=0$ and $\Gamma=A$.

Figure 3 shows the variations of the electric field and of the average value $\left\langle\Phi_{Z x}\right\rangle$. The electric field displays slow and small variations from 0 to $30 \mathrm{ps}$ and faster and larger variations between 30 and 50 ps. For $\left\langle\Phi_{Z x}\right\rangle$, a nearly periodic behavior can be seen from 3 to 20 ps. The windowed Fourier transform of the electric field is shown in Fig. 4. This figure displays a limited number of horizontal features characterized by wavenumbers ranging from 15 to $155 \mathrm{~cm}^{-1}$. These correspond to frequency components of the electric field and the wavenumbers of the lowest components are $15,23,33$, and $43 \mathrm{~cm}^{-1}$. Table IV, where the field-free strongest electric dipole rotational transitions involving $M=0, A_{e}$-type levels are listed up to $160 \mathrm{~cm}^{-1}$, allows us to assign these wavenumbers in terms of transitions. The wavenumbers of 15 , 23 , and $33 \mathrm{~cm}^{-1}$ can be unambiguously assigned to the $1_{11} \leftarrow 0_{00}, 2_{02} \leftarrow 1_{11}$, and $3_{13} \leftarrow 2_{02}$ transitions, respectively. There is no obvious assignment for the transition at $43 \mathrm{~cm}^{-1}$ which can either be the $4_{04} \leftarrow 3_{13}$ or the $2_{20} \leftarrow 1_{11}$ transition. Figure 4 also shows that the frequency components do not appear at the same time. The transition at $15 \mathrm{~cm}^{-1}$ can be seen from 2 to $25 \mathrm{ps}$ while the one at $23 \mathrm{~cm}^{-1}$ can be seen later, from 8 to $42 \mathrm{ps}$. The former transition ensures that the terahertz pulse first populates the $1_{11}$ level and, once it is enough populated, the $2_{02}$ level start being populated with the second transition. The $33 \mathrm{~cm}^{-1}$ wavenumber appearing between 24 and $47 \mathrm{ps}$ enables the terahertz pulse to populate the $3_{13}$ level since the $2_{02}$ is populated for $t>24$ ps. The transition at $43 \mathrm{~cm}^{-1}$ can now be unambiguously assigned as the $2_{20} \leftarrow 1_{11}$ transition since it starts appearing at $t=8 \mathrm{ps}$ when the $1_{11}$ level is populated but the $3_{13}$ level 


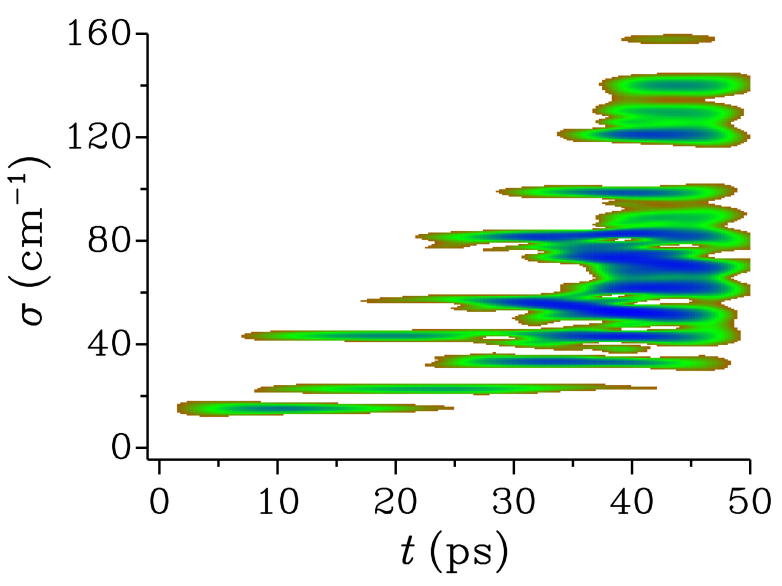

Figure 4. Windowed Fourier transform of the optimized electric field obtained in Section III B 1 for a zero temperature. The $x$ and $y$ axes are the time and the frequency in picosecond and $\mathrm{cm}^{-1}$, respectively. A darker color indicates larger values of the squared Fourier transform modulus.

Table IV. Strongest $M=0, A_{e}$-type transitions ${ }^{\mathrm{a}}$

\begin{tabular}{cccccccc}
\hline \hline$J_{K_{a} K_{c}}$ & $J_{K_{a} K_{c}}$ & $\sigma^{\mathrm{b}}$ & $S^{\mathrm{c}}$ & $J_{K_{a} K_{c}}$ & $J_{K_{a} K_{c}}$ & $\sigma^{\mathrm{b}}$ & $S^{\mathrm{c}}$ \\
\hline $1_{11}$ & $0_{00}$ & 15.090 & 0.32 & $6_{24}$ & $5_{33}$ & 80.525 & 0.13 \\
$2_{02}$ & $1_{11}$ & 22.926 & 0.16 & $4_{40}$ & $3_{31}$ & 81.462 & 0.16 \\
$3_{13}$ & $2_{02}$ & 33.449 & 0.20 & $9_{19}$ & $8_{08}$ & 89.839 & 0.22 \\
$4_{04}$ & $3_{13}$ & 42.707 & 0.21 & $7_{35}$ & $6_{24}$ & 90.604 & 0.15 \\
$2_{20}$ & $1_{11}$ & 43.279 & 0.09 & $5_{51}$ & $4_{40}$ & 99.302 & 0.19 \\
$5_{15}$ & $4_{04}$ & 52.172 & 0.21 & $8_{26}$ & $7_{35}$ & 99.767 & 0.16 \\
$3_{31}$ & $2_{20}$ & 56.972 & 0.17 & $9_{37}$ & $8_{26}$ & 109.126 & 0.17 \\
$6_{06}$ & $5_{15}$ & 61.601 & 0.22 & $7_{53}$ & $6_{42}$ & 117.938 & 0.09 \\
$7_{17}$ & $6_{06}$ & 71.025 & 0.22 & $6_{60}$ & $5_{51}$ & 120.742 & 0.19 \\
$5_{33}$ & $4_{22}$ & 74.057 & 0.12 & $9_{55}$ & $8_{44}$ & 129.902 & 0.11 \\
$8_{08}$ & $7_{17}$ & 80.438 & 0.22 & $7_{71}$ & $6_{60}$ & 140.060 & 0.20 \\
\hline \hline
\end{tabular}

a Transitions are assigned using the rotational quantum numbers $J, K_{a}$, and $K_{c}$ of the upper and lower $M=0, A_{e}$-type levels.

b Transition wavenumber in $\mathrm{cm}^{-1}$.

c Transition strength in Debye ${ }^{2}$ for $M=0$.

is not. The other frequency components in Fig. 4 can be similarly interpreted. The population is transferred from the $0_{00}$ level to higher lying level by a mechanism quite analogous to the rotational ladder climbing theoretically predicted for linear molecules by Salomon et al. ${ }^{26}$

At time $T$, the wavefunction of the molecule can be expanded in terms of $M=0, A_{e}$-type eigenfunctions of $\Phi_{Z x}$ associated with the largest eigenvalues. The wavefunction is $77.6,1.8,15.7,0.7$, and $2.9 \%$ of the eigenfunctions associated with the nondegenerate eigenvalues $0.97391,0.88141,0.86506,0.69206$, and 0.67941 , respectively. These 5 eigenfunctions account for $98.8 \%$ of the wavefunction.
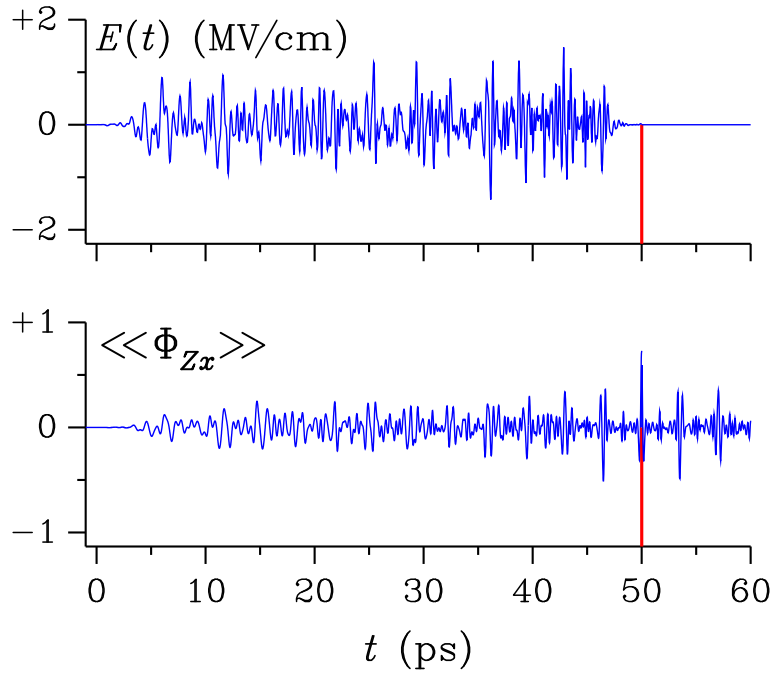

Figure 5. The optimized electric field $E(t)$ obtained for a temperature of $50 \mathrm{~K}$ in Section III B 2 (top panel) and the thermal average $\left\langle\left\langle\Phi_{Z x}\right\rangle\right\rangle$ (lower panel) as a function of the time $t$ in ps. For the optimization time $T=50 \mathrm{ps}$, indicated by a vertical line in both panels, $\left\langle\left\langle\Phi_{Z x}\right\rangle\right\rangle=0.74230$.

\section{Temperature of $50 \mathrm{~K}$}

At $t=0$, the density matrix of the molecule is $\rho_{\text {boltz }}(\mathcal{T})$, the density matrix describing a Boltzmannian equilibrium characterized by a temperature $\mathcal{T}=50 \mathrm{~K}$. When $t>0$, the density matrix is propagated using the time evolution operator; each $M$ value and $C_{2}$ symmetry species being propagated independently. Taking $J_{\mathrm{Max}}=13$, convergence was reached after 700 iterations and the thermal average of $\Phi_{Z x}$ was 0.74230 . This number should be compared to 0.93014 , the value of $\left\langle\left\langle\Phi_{Z x}\right\rangle\right\rangle_{\text {Max }}$ for a temperature of $50 \mathrm{~K}$.

Figure 5 shows the variations of the electric field and of $\left\langle\left\langle\Phi_{Z x}\right\rangle\right\rangle$ the thermal average of $\Phi_{Z x}$. Fast variations of the electric field can be seen throughout the pulse. Its windowed Fourier transform is displayed in Fig. 6. A limited number of frequency components can be observed, as in the case of the zero temperature, but their number is larger. For instance a wavenumber of $5 \mathrm{~cm}^{-1}$ can clearly be seen in the present figure and has no counterpart in Figure 3. Using the table given as supplementary material, where all the allowed transitions are listed up to $160 \mathrm{~cm}^{-1}$, allows us to assign the wavenumbers in Fig. 6 . It is found that the additional wavenumbers are those of transitions that are not allowed for a zero temperature because they either do not appear for $M=0$, like the transition at $5 \mathrm{~cm}^{-1}$ which turns out to be the $Q$-type $1_{10} \leftarrow 1_{01}$ transition, or they involve levels belonging to the $B$ symmetry species of $C_{2}$. Figure 6 also shows that most frequency components start appearing in the beginning of the pulse, unlike in the zero temperature case. In the case of a finite temperature, low lying rotational levels are already populated and the terahertz pulse is 


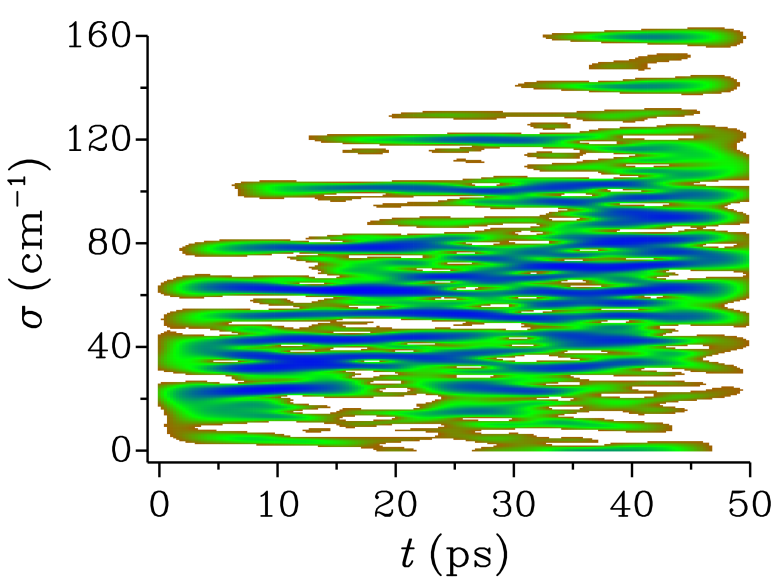

Figure 6. Windowed Fourier transform of the optimized electric field obtained in Section III B 2 for a temperature of $50 \mathrm{~K}$. The $x$ and $y$ axes are the time and the frequency in picosecond and $\mathrm{cm}^{-1}$, respectively. A darker color indicates larger values of the squared Fourier transform modulus.

able to change their population.

\section{OPTIMIZED LASER PULSE}

The laser field, taken polarized along the laboratoryfixed $Z$ axis, is written $\mathcal{E}(t)=E(t) \cos \omega t \mathbf{i}_{Z}$, where $\omega$ is the angular frequency of the laser and $E(t)$ is the laser field envelope. The latter is optimized so as to maximize the alignment of the molecule-fixed $y$ axis parallel to the laboratory-fixed $Z$ axis. The time-dependent Hamiltonian describing the molecule includes the quadratic coupling term of the Stark coupling Hamiltonian in Eq. (2):

$$
H(t)=H_{\mathrm{R}}-\frac{E(t)^{2}}{4} \sum_{\gamma=x, y, z} \alpha_{\gamma \gamma} \Phi_{Z \gamma}^{2}
$$

where $\Phi_{Z \gamma}^{2}$, with $\gamma=x, y, z$, are squared direction cosines. In this equation, the $1 / 4$ factor replaces the $1 / 2$ factor in Eq. (2) because Eq. (16), obtained after time averaging over the laser period, is expressed in terms of the laser field envelope. The Hamiltonian in Eq. (16) belongs to the completely symmetrical $A_{1}$ symmetry species of $C_{2 v}$. Its matrix with the $|J, k, M\rangle$ basis set functions of Section II A can be split into the same number of submatrices, with the same dimensions, as the Hamiltonian in Eq. (8). Each submatrix is identified by $M$ and can be split into four blocks corresponding to the four symmetry species of $C_{2 v}$. Each block is of $p \times p$ dimensions where $p$ is $p(\Gamma,|M|)$. For $M=0$, each block can be further split into two sub-blocks because the Stark coupling operator in the Hamiltonian of Eq. (16) then only displays nonvanishing $|\Delta J|=0, \pm 2$ matrix elements. The resulting eight sub-blocks can be identified using the symmetry species of $C_{2 v}$ and the parity of $J$.

\section{A. Monotonically convergent algorithm}

The monotonically convergent algorithm of Lapert et $a l .{ }^{21}$ is based on a target state described by its density matrix. Using the notation of the present paper and remembering that the $C_{2 v}$ group should be utilized, this density matrix becomes:

$$
\rho_{\mathrm{opt}}=\sum_{\Gamma} \sum_{M=-J_{\mathrm{Max}}}^{+J_{\mathrm{Max}}} \sum_{k=1}^{p(\Gamma,|M|)} \omega_{n(k)}^{\Gamma, J(k)}\left|\phi_{k}^{M, \Gamma}\right\rangle\left\langle\phi_{k}^{M, \Gamma}\right|,
$$

where $\Gamma$ spans the four symmetry species of $C_{2 v} ; \omega_{n}^{\Gamma, J}$ is the Boltzmann factor for the field-free rotational level characterized by a $C_{2 v}$ symmetry species $\Gamma$, a rotational quantum number $J \geq|M|$, and the index $n$; and $\left|\phi_{k}^{M, \Gamma}\right\rangle$ is an eigenfunction of $\Phi_{Z y}^{2}$. As in Eq. (13), the one to one correspondence between $J, n$ and $k$ allows us to choose the functions $J(k)$ and $n(k)$ so that the lowest lying field-free levels are associated with the largest eigenvalues. This choice leads to the largest value of the thermal average of $\Phi_{Z y}^{2}$. In the algorithm of Lapert et al., ${ }^{21}$ a density matrix $\rho(t)$ and its adjoint $\chi(t)$ are also used. Both satisfy the von Neumann equation with the Hamiltonian in Eq. (16) and are propagated forward and backward with initial and final conditions $\rho(t=0)=\rho_{\text {boltz }}(\mathcal{T})$ and $\chi(T)=\rho_{\text {opt }}$, respectively.

In the present optimization, a cost function which is quartic in the electric field is adopted. ${ }^{21}$ In the equations of this reference allowing us to update the laser field envelope, the matrix elements $\mu_{p, q}$ and $\beta_{p, q}$, corresponding to the permanent dipole moment and the hyperpolarizability, are ignored and the matrix element $\alpha_{p, q}$, corresponding to the polarizability, is rewritten:

$$
\alpha_{p, q}=-\frac{i}{2} \operatorname{Tr}\left\{\rho^{(q)}(t)\left[\chi^{(p)}(t), \sum_{\gamma=x, y, z} \alpha_{\gamma \gamma} \Phi_{Z \gamma}^{2}\right]\right\}
$$

where $\rho^{(q)}(t)$ and $\chi^{(p)}(t)$ are the density matrix and its adjoint computed for the laser field envelopes $E^{(q)}(t)$ and $E^{(p)}(t)$ at the $q$ th and $p$ th iterations, respectively.

To determine $\rho_{\text {opt }}$, eigenvalues and eigenfunctions of $\Phi_{Z y}^{2}$ should be computed. Setting up its matrix with the $|J, k, M\rangle$ basis set functions of Section II A and using the results in Section II B yields a matrix which can be block-diagonalized in the same way as the Hamiltonian in Eq. (16). Figure 7 shows $\lambda_{\text {Max }}^{M, \Gamma}$ the largest eigenvalues of $\Phi_{Z y}^{2}$ for $J_{\mathrm{Max}}=9,0 \leq M \leq J_{\mathrm{Max}}$, and for all four symmetry species of $C_{2 v}$. These largest eigenvalues are doubly degenerate for $|M| \geq 2$. For $|M|<2$, this is only the case for the $B_{1}$ and $B_{2}$ symmetry species. As in the case of the direction cosine $\Phi_{Z x}$, there is a fast variation with the parity of $M$ superimposed on a slower decrease with $M$. For an even (odd) value of $M$, the eigenvalues for $\Gamma=A_{1}$ and $A_{2}$ are larger (smaller) than those for $\Gamma=B_{1}$ and $B_{2}$. The largest eigenvalue of $\Phi_{Z y}^{2}$ is obtained for $M=0$ and $\Gamma=A_{1}\left(\Gamma=A_{2}\right)$ when $J_{\text {Max }}$ is even (odd); the corresponding eigenfunction involves 


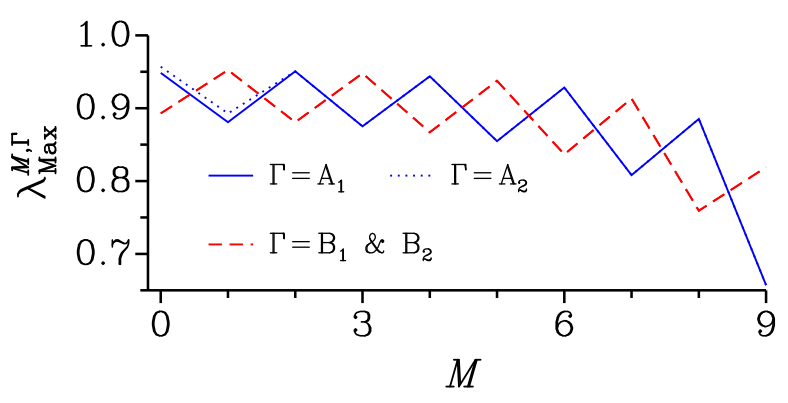

Figure 7. The largest eigenvalue $\lambda_{\mathrm{Max}}^{M, \Gamma}$ of the squared direction cosine $\Phi_{Z y}^{2}$ as a function of $M$ for $J_{\text {Max }}=9$. Solid and dotted lines correspond to the $C_{2 v}$ symmetry species $A_{1}$ and $A_{2}$, respectively. A dashed line corresponds to the $C_{2 v}$ symmetry species $B_{1}$ and $B_{2}$.

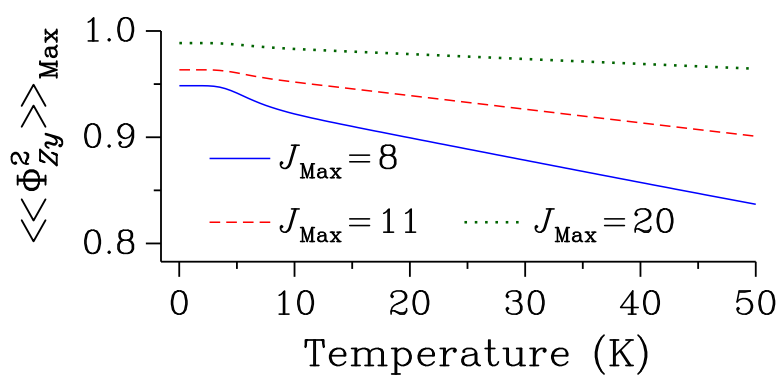

Figure 8. The maximum alignment $\left\langle\left\langle\Phi_{Z_{y}}^{2}\right\rangle\right\rangle_{\text {Max }}$, calculated with Eq. (19), is plotted as a function of the temperature in Kelvin in solid, dashed, and dotted lines, for $J_{\mathrm{Max}}=8,11$, and 20 , respectively.

rotational functions with $J$ even (odd). For a given $C_{2 v}$ symmetry species $\Gamma$ and a given $M$ value, the direction cosine $\Phi_{Z y}^{2}$ only displays nondegenerate eigenvalues. This allows us to unambiguously assign a field-free rotational level to an eigenvalue of $\Phi_{Z y}^{2}$ in Eq. (17).

The maximum alignment for a given temperature $\mathcal{T}$ can be estimated evaluating the thermal average of $\Phi_{Z y}^{2}$ with the target state $\rho_{\text {opt }}$. This leads to:

$$
\left\langle\left\langle\Phi_{Z y}^{2}\right\rangle\right\rangle_{\mathrm{Max}}=\sum_{\Gamma} \sum_{M=-J_{\mathrm{Max}}}^{+J_{\mathrm{Max}}} \sum_{k=1}^{p(\Gamma,|M|)} \omega_{n(k)}^{\Gamma, J(k)} \lambda_{k}^{M, \Gamma},
$$

where $\Gamma$ spans the four symmetry species of $C_{2 v}$. Figure 8 shows the variations of $\left\langle\left\langle\Phi_{Z y}^{2}\right\rangle\right\rangle_{\text {Max }}$ with the temperature for several values of $J_{\text {Max }}$. As in the case of the largest orientation, taking a larger $J_{\text {Max }}$ leads to a larger maximum alignment for a given temperature. For a given value of $J_{\mathrm{Max}}$, the maximum alignment decreases with the temperature.

\section{B. Numerical results}

In the iterative procedure, ${ }^{21}$ the optimization time $T$ was set to $5 \mathrm{ps}$ for the zero temperature, to $6 \mathrm{ps}$ for the temperature of $20 \mathrm{~K}$, and 1024 time intervals of equal duration were used for the computation of the time evolution of the density matrix and its adjoint. The penalty factor $\lambda$ in the cost functional of Eq. (3) of Lapert et al. ${ }^{21}$ was written:

$$
\lambda(t)=\frac{\lambda_{0}}{\sin ^{2}(\pi t / T)},
$$

where $\lambda_{0}$ was set to $3.7 \times 10^{-7}$ for the zero temperature and to $1.8 \times 10^{-7}$ for the temperature of $20 \mathrm{~K}$, expressing the laser field envelope in $\mathrm{kV} / \mathrm{cm}$ units. The initial laser field envelope $E^{(0)}(t)$ was set to a small constant value. Equations (24) and (25) of Lapert et al. ${ }^{21}$ were solved to update the laser field envelope after the backward and forward propagations, respectively. In these equations, $\eta_{1}$ and $\eta_{2}$ were set to 1 .

The values chosen for $\lambda_{0}$ in Eq. (20) lead to maximum intensities on the order of $2 \times 10^{14} \mathrm{~W} / \mathrm{cm}^{2}$ for the designed laser pulses. Such a large value ensures, at least for the zero temperature, a significant alignment with $\left\langle\Phi_{Z y}^{2}\right\rangle>0.9$. Large intensities arise because $\alpha_{y y}$, the largest component of the polarizability tensor, is only 0.114 and $0.092 \AA^{3}$ larger than its two other components, $\alpha_{x x}$ and $\alpha_{z z}$, respectively, and a very large electric field is required to interact efficiently with the molecule. It should be kept in mind that an intensity of $2 \times 10^{14} \mathrm{~W} / \mathrm{cm}^{2}$ may be close or even larger than the offsets of both tunnel and multiphoton ionization. A maximum intensity on the order of $5 \times 10^{13} \mathrm{~W} / \mathrm{cm}^{2}, 4$ times smaller and physically more satisfactory, can be obtained setting $\lambda_{0}$ in Eq. $(20)$ to $4 \times 10^{-6}$ and $8.5 \times 10^{-7}$ for the zero and $20 \mathrm{~K}$ temperatures, respectively. Much smaller alignments are then reached for both temperatures.

\section{Zero temperature}

For a zero temperature, only one term arises in the density matrix $\rho(t)$, in its adjoint $\chi(t)$, and in the target state $\rho_{\text {opt }}$. The latter is simply $\left|\phi_{\text {Max }}^{M, \Gamma}\right\rangle\left\langle\phi_{\text {Max }}^{M, \Gamma}\right|$, where $\left|\phi_{\mathrm{Max}}^{M, \Gamma}\right\rangle$ is the eigenfunction of the largest eigenvalue of $\Phi_{Z y}^{2}$ for $M=0$ and the $A_{1}$ symmetry. The density matrix and its adjoint reduce to $|\psi(t)\rangle\langle\psi(t)|$ and $|\phi(t)\rangle\langle\phi(t)|$, respectively. For $t=0,|\psi(t)\rangle$ is the $A_{1}$ symmetry wavefunction of the $0_{00}, M=0$ ground rotational level; for $t=T,|\phi(t)\rangle$ is $\left|\phi_{\mathrm{Max}}^{M, \Gamma}\right\rangle$. Both $|\psi(t)\rangle$ and $|\phi(t)\rangle$ remain $A_{1}$ symmetry wavefunctions due to the block decomposition of the time evolution operator. Taking $J_{\mathrm{Max}}=11$, convergence was reached after 400 iterations and the average values of $\Phi_{Z x}^{2}, \Phi_{Z y}^{2}$, and $\Phi_{Z z}^{2}$ at the end of the pulse were $0.02093,0.94416$, and 0.03491 , respectively. The average value of $\Phi_{Z y}^{2}$ should be compared to 0.96346 the value of $\lambda_{\text {Max }}^{M, \Gamma}$ for $M=0$ and the $A_{1}$ symmetry species.

Figure 9 depicts the variations of the intensity of the laser pulse and of the average value $\left\langle\Phi_{Z y}^{2}\right\rangle$. The laser pulse consists of a series of short kick pulses with a duration of approximately $0.1 \mathrm{ps}$; the intensity of the strongest pulse is $1.8 \times 10^{14} \mathrm{~W} / \mathrm{cm}^{2}$. The average value 

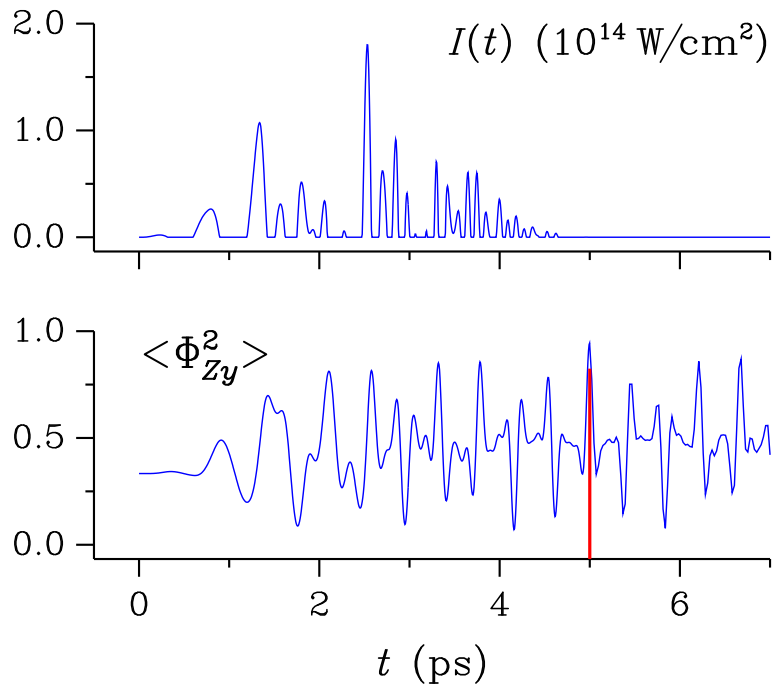

Figure 9. The intensity of the optimized laser pulse $I(t)$ obtained for a zero temperature in Section IV B 1 (top panel) and the average value $\left\langle\Phi_{Z y}^{2}\right\rangle$ (lower panel) as a function of the time $t$ in ps. For the optimization time $T=5 \mathrm{ps}$, indicated by a vertical line in the lower panel, $\left\langle\Phi_{Z y}^{2}\right\rangle=0.94416$.

$\left\langle\Phi_{Z y}^{2}\right\rangle$ is $1 / 3$ for $t=0$ and displays smooth variations between 0 and 2 ps. Insight into the mechanism by which the laser pulse interacts with the molecule can be retrieved computing the average values of the operators $J_{z}^{2}$ and $J_{x}^{2}+J_{y}^{2}$ containing information about the nature of the rotational levels involved in the expansion of the wavefunction. Figure 10 displays the variation of the average values $\left\langle J_{z}^{2}\right\rangle$ and $\left\langle J_{x}^{2}+J_{y}^{2}\right\rangle$. Both increase almost monotonically with time during the pulse. After the pulse, small variations of $\left\langle J_{z}^{2}\right\rangle$ and $\left\langle J_{x}^{2}+J_{y}^{2}\right\rangle$ can still be observed because neither $J_{z}^{2}$ nor $J_{x}^{2}+J_{y}^{2}$ commute with the field-free Hamiltonian. Examining Fig. 9 shows that both averages increase significantly with each kick pulse of the laser. This can clearly be seen at $t=1.3$ and $2.5 \mathrm{ps}$. At the end of the pulse, the larger value of $\left\langle J_{z}^{2}\right\rangle$ compared to $\left\langle J_{x}^{2}+J_{y}^{2}\right\rangle$ and the smallness of $\left\langle\Phi_{Z x}^{2}\right\rangle$ and $\left\langle\Phi_{Z z}^{2}\right\rangle$ are consistent with the molecule rotating mainly about its molecule-fixed $z$ axis. The laser pulse thus populates high lying rotational characterized by a large $K_{a}$ value.

At time $T$, the wavefunction of the molecule can be expanded in terms of $M=0, A_{1}$-type eigenfunctions of $\Phi_{Z y}^{2}$ involving rotational levels with $J$ even and associated with the largest eigenvalues. The wavefunction is 89.1, $4.4,5.2$, and $0.6 \%$ of the eigenfunctions associated with the nondegenerate eigenvalues $0.96346,0.83926,0.81743$, and 0.59275 , respectively. These 4 eigenfunctions account for $99.3 \%$ of the wavefunction. Although the initial laser field envelope $E^{(0)}(t)$ is not quite satisfactory from the physical point of view, Fig. 9 emphasizes that the optimized laser field envelope displays the correct behavior as it vanishes at the beginning and the end of the laser pulse.

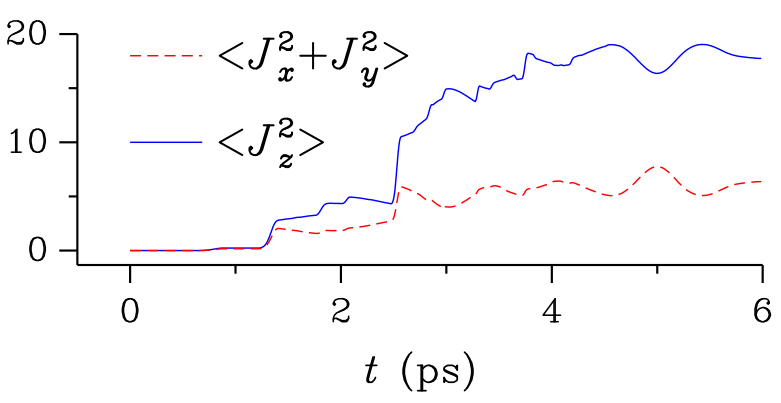

Figure 10. The average values $\left\langle J_{z}^{2}\right\rangle$ and $\left\langle J_{x}^{2}+J_{y}^{2}\right\rangle$ as a function of the time $t$ in ps in solid and dashed lines, respectively, for the optimized laser pulse obtained in Section IV B 1 for a zero temperature.

Selecting a maximum intensity 4 times smaller, equal to $5 \times 10^{13} \mathrm{~W} / \mathrm{cm}^{2}$, leads to a much smaller alignment with the average values of $\Phi_{Z x}^{2}, \Phi_{Z y}^{2}$, and $\Phi_{Z z}^{2}$ being $0.069303,0.825396$, and 0.105301 , respectively, at the end of the pulse.

\section{Temperature of $20 \mathrm{~K}$}

For a nonzero temperature, both the density matrix and its adjoint are propagated using the initial conditions in Section IV A for $\mathcal{T}=20 \mathrm{~K}$. Due to the block decomposition of the evolution operator, each $M$ value and $C_{2 v}$ symmetry species can be propagated independently. Taking $J_{\mathrm{Max}}=11$, convergence was reached after 500 iterations and the thermal averages of $\Phi_{Z x}^{2}, \Phi_{Z y}^{2}$, and $\Phi_{Z z}^{2}$ at the end of the pulse were 0.13991, 0.71720, and 0.14289 , respectively. The thermal average of $\Phi_{Z y}^{2}$ should be compared to 0.93921 the value of $\left\langle\left\langle\Phi_{Z y}^{2}\right\rangle\right\rangle_{\text {Max }}$ for a temperature of $20 \mathrm{~K}$.

Figure 11 shows the variations of the intensity of the laser pulse and of the thermal average $\left\langle\left\langle\Phi_{Z y}^{2}\right\rangle\right\rangle$. Just as for the zero temperature, the laser pulse consists of a series of short kick pulses with a duration of approximately $0.1 \mathrm{ps}$; the intensity of the strongest pulse is $1.9 \times 10^{14} \mathrm{~W} / \mathrm{cm}^{2}$. Taking a maximum intensity 4 times smaller, close to $5 \times$ $10^{13} \mathrm{~W} / \mathrm{cm}^{2}$, leads to a much smaller alignment with the thermal averages of $\Phi_{Z x}^{2}, \Phi_{Z y}^{2}$, and $\Phi_{Z z}^{2}$ being respecively $0.228479,0.529576$, and 0.241945 at the end of the pulse.

\section{DISCUSSION}

Although optimal control theory has already been applied to the orientation and alignment of linear molecules, ${ }^{22,26}$ it has not been applied yet to asymmetrictop molecules. The present paper reports on the application of control theory to the optimal orientation and alignment of such molecules with terahertz and laser pulses. The theoretical treatment presented is suited for any asymmetric-top molecule displaying a permanent 

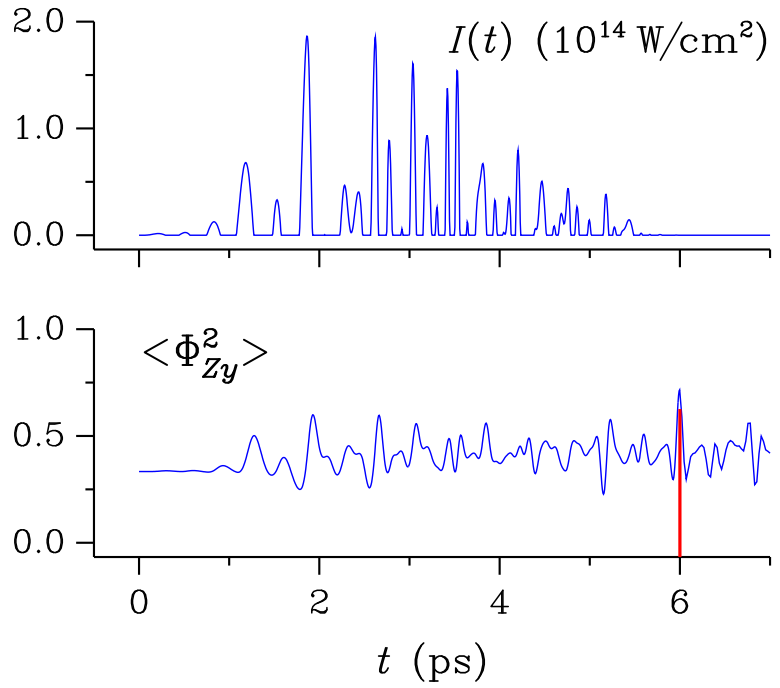

Figure 11. The intensity of the optimized laser pulse $I(t)$ obtained for a temperature of $20 \mathrm{~K}$ in Section IV B 2 (top panel) and the thermal average $\left\langle\left\langle\Phi_{Z y}^{2}\right\rangle\right\rangle$ (lower panel) as a function of the time $t$ in ps. For the optimization time $T=6 \mathrm{ps}$, indicated by a vertical line in the lower panels, $\left\langle\left\langle\Phi_{Z y}^{2}\right\rangle\right\rangle=0.71720$.

dipole moment or large and different enough polarizability tensor components. In the present paper, it is applied to the asymmetric-top $\mathrm{H}_{2} \mathrm{~S}$ molecule. Its $C_{2 v}$ symmetry permits a block diagonalization of the various operators involved in the calculation, reducing computing time. The large rotational constants of the $\mathrm{H}_{2} \mathrm{~S}$ molecule also allows us to obtain converged numerical results with a maximum value of $J$ smaller than 15 , even for a temperature of $50 \mathrm{~K}$, further reducing computing time. The theoretical treatment could be used with other $C_{2 v}$ symmetry asymmetric-top molecule like, for instance, the formaldehyde $\left(\mathrm{H}_{2} \mathrm{CO}\right)$ molecule. In this molecule, as the dipole moment lies along the molecule-fixed $z$ axis and the largest component of the polarizability tensor is $\alpha_{z z}$ (using the $\mathrm{I}^{r}$ representation), the orientation and alignment will be qualitatively different. ${ }^{43}$ In the case of $C_{s}$ or $C_{1}$ symmetry molecules, the theoretical treatment should be modified to account for the fact that a block diagonalization of the operators involved in the calculation is not possible as the dipole moment is not parallel to either principal axes of inertia. Such would be the case for the $C_{s}$ symmetry vinyl chloride $\left(\mathrm{H}_{2} \mathrm{CCHCl}\right)$ molecule with a dipole moment neither parallel to the $a$ nor to the $b$ principal axes.

Using the TBQCP of Ho and Rabitz ${ }^{23}$ and its extended version for mixed states of Liao et al. ${ }^{25}$ terahertz pulses were designed to maximize the orientation of the molecule-fixed $x$ axis along the laboratoryfixed $Z$ axis. The electric field was optimized iteratively making use of an update relation based on Eq. (11) of the present paper. This equation involves the positive semidefinite operator $O(t)$ satisfying the invariant equation introduced by Ho and Rabitz. ${ }^{23}$ In the present case, it is expressed in terms of the eigenvalues and eigenfunctions of the direction cosine $\Phi_{Z x}$, as appropriate for an asymmetric-top molecule. A zero and a finite temperatures of $50 \mathrm{~K}$ were considered and the time variation of the optimized electric field is displayed in Figs. 3 and 5. Both pulses are $50 \mathrm{ps}$ long and the maximum value of the electric field is less than $2 \mathrm{MV} / \mathrm{cm}$. For the zero temperature the orientation achieved $\left\langle\Phi_{Z x}\right\rangle=0.95973$ compares favorably with the maximum theoretical orientation, $\left\langle\Phi_{Z x}\right\rangle_{\text {Max }}=0.97391$. For the finite temperature, the designed terahertz pulse is not as effective and the orientation obtained $\left\langle\left\langle\Phi_{Z x}\right\rangle\right\rangle=0.74230$ is below that for the zero temperature and for the maximum theoretical orientation $\left\langle\left\langle\Phi_{Z x}\right\rangle\right\rangle_{\mathrm{Max}}=0.93014$. For the zero temperature, the mechanism by which the terahertz pulse populates high-lying rotational levels, starting from the $J=0$ ground rotational level, is a rotational ladder climbing during which population is transferred between levels connected by electric dipole transitions. This mechanism is analogous to the one evidenced in linear molecules. ${ }^{26}$ For the finite temperature, a similar mechanism takes place, but several levels are populated before the pulse.

The monotonically convergent algorithm of Lapert et $a l^{21}$ was utilized to build laser pulses maximizing the alignment of the molecule-fixed $y$ axis parallel to the laboratory-fixed $Z$ axis. This algorithm also leads to an iterative process and is based on a target state expressed in term of its density matrix. The latter, initially written for a linear molecule, ${ }^{21}$ was modified for an asymmetric-top molecule and appears in Eq. (17) of the present paper. It involves the eigenvalues and eigenfunctions of the squared direction cosine $\Phi_{Z y}^{2}$, computed for this type of molecule. A zero temperature and a finite temperature of $20 \mathrm{~K}$ were considered and the time variation of the intensity of the laser pulse can be seen in Figs. 9 and 11. For both temperatures, the laser pulse consists of a series of kick pulses. This result is similar to that derived by Lapert et $a l^{22}$ for the alignment of $\mathrm{CO}$ molecules without spectral constraints. For the zero temperature, the alignment achieved in the present investigation with a $5 \mathrm{ps}$ laser pulse, $\left\langle\Phi_{Z y}^{2}\right\rangle=0.94416$, is quite close the maximum theoretical one. For the finite temperature, although a longer 6 ps long laser pulse was built, the alignment achieved $\left\langle\left\langle\Phi_{Z y}^{2}\right\rangle\right\rangle=0.71720$ is well below the maximum theoretical one.

Although the irregular and incommensurable spacings of the rotational energy levels of an asymmetrictop molecules might reduce the effectiveness of control theory, ${ }^{44}$ the present paper shows that, at least for a zero temperature, it is as effective for this type of molecule as in the simpler case of linear molecules.

\section{SUPPLEMENTARY MATERIAL}

See supplementary material for a PDF file containing two tables. The spectroscopic constants for the ground vibrational state of $\mathrm{H}_{2} \mathrm{~S}$ appear in the first one and the 
[1] H. Stapelfeldt and T. Seideman, Rev. Mod. Phys. 75, 543 (2003).

[2] T. Seideman and E. Hamilton, in Advances In Atomic, Molecular, and Optical Physics, Vol. 52, edited by P. Berman and C. Lin (Academic Press, 2005) pp. 289329.

[3] D. Normand, L. A. Lompré, and C. Cornaggia, J. Phys. B 25, L497 (1992).

[4] B. Friedrich and D. Herschbach, Phys. Rev. Lett. 74, 4623 (1995).

[5] H. Sakai, C. P. Safvan, J. J. Larsen, K. M. Hilligsøe, K. Hald, and H. Stapelfeldt, J. Chem. Phys. 110, 10235 (1999).

[6] C.-C. Shu, K.-J. Yuan, W.-H. Hu, and S.-L. Cong, J. Chem. Phys. 132, 244311 (2010).

[7] S. Fleischer, Y. Zhou, R. W. Field, and K. A. Nelson, Phys. Rev. Lett. 107, 163603 (2011).

[8] P. Babilotte, K. Hamraoui, F. Billard, E. Hertz, B. Lavorel, O. Faucher, and D. Sugny, Phys. Rev. A 94, 043403 (2016).

[9] O. Ghafur, A. Rouzée, A. Gijsbertsen, W. K. Siu, S. Stolte, and M. J. J. Vrakking, Nat. Phys. 5, 289 (2009).

[10] S. De, I. Znakovskaya, D. Ray, F. Anis, N. G. Johnson, I. A. Bocharova, M. Magrakvelidze, B. D. Esry, C. L. Cocke, I. V. Litvinyuk, and M. F. Kling, Phys. Rev. Lett. 103, 153002 (2009).

[11] H. Sakai, S. Minemoto, H. Nanjo, H. Tanji, and T. Suzuki, Phys. Rev. Lett. 90, 083001 (2003).

[12] A. Goban, S. Minemoto, and H. Sakai, Phys. Rev. Lett. 101, 013001 (2008).

[13] D. Takei, J. H. Mun, S. Minemoto, and H. Sakai, Phys. Rev. A 94, 013401 (2016).

[14] K. Kitano, N. Ishii, and J. Itatani, Phys. Rev. A 84, 053408 (2011).

[15] F. Weise, S. M. Weber, M. Plewicki, and A. Lindinger, Chem. Phys. 332, 313 (2007).

[16] A. M. Weiner, Opt. Commun. 284, 3669 (2011).

[17] M. Sato, T. Higuchi, N. Kanda, K. Konishi, K. Yoshioka, T. Suzuki, K. Misawa, and M. Kuwata-Gonokami, Nature Photon. 7, 724 (2013).

[18] G. Turinici and H. Rabitz, Phys. Rev. A 70, 063412 (2004).

[19] M. Mirrahimi, G. Turinici, and P. Rouchon, J. Phys. Chem. A 109, 2631 (2005).
[20] J. Werschnik and E. K. U. Gross, J. Phys. B 40, R175 (2007).

[21] M. Lapert, R. Tehini, G. Turinici, and D. Sugny, Phys. Rev. A 78, 023408 (2008).

[22] M. Lapert, R. Tehini, G. Turinici, and D. Sugny, Phys. Rev. A 79, 063411 (2009).

[23] T.-S. Ho and H. Rabitz, Phys. Rev. E 82, 026703 (2010).

[24] T.-S. Ho, H. Rabitz, and S.-I. Chu, Comp. Phys. Commun. 182, 14 (2011).

[25] S.-L. Liao, T.-S. Ho, H. Rabitz, and S.-I. Chu, Phys. Rev. A 87, 013429 (2013).

[26] J. Salomon, C. M. Dion, and G. Turinici, J. Chem. Phys. 123, 144310 (2005)

[27] M. Artamonov and T. Seideman, Phys. Rev. A 82, 023413 (2010).

[28] L. H. Coudert, J. Chem. Phys. 146, 024303 (2017).

[29] A. Rouzée, S. Guérin, V. Boudon, B. Lavorel, and O. Faucher, Phys. Rev. A 73, 033418 (2006).

[30] K. M. T. Yamada and S. Klee, J. Mol. Spectrosc. 166, 395 (1994).

[31] R. Viswanathan and T. R. Dyke, J. Mol. Spectrosc. 103, 231 (1984).

[32] J. M. Fernández-Sánchez and W. F. Murphy, J. Mol. Spectrosc. 156, 444 (1992).

[33] P. R. Bunker, Molecular Symmetry and Spectroscopy, 1st ed. (Academic Press, New York, 1979).

[34] J. K. G. Watson, J. Chem. Phys. 46, 1935 (1967).

[35] J. K. G. Watson, J. Chem. Phys. 48, 181 (1968).

[36] J. K. G. Watson, J. Chem. Phys. 48, 4517 (1968).

[37] E. P. Wigner, Group Theory (Academic Press, New York, 1959).

[38] K. K. Svidzinskii, Theory of the Hyperfine Structure of the Rotational Spectra of Molecules, edited by D. V. Skobel'tsyn (Soviet Maser Research, 1964) pp. 88-148.

[39] S. C. Wang, Phys. Rev. 34, 243 (1929).

[40] L. H. Coudert, J. Mol. Spectrosc. 132, 13 (1988).

[41] A. R. Edmonds, Angular Momentum in Quantum Mechanics (Princeton University Press, Princeton, 1960).

[42] H. R. Lewis, Jr. and W. B. Riesenfeld, J. Math. Phys. 10, 1458 (1969).

[43] Z. Benková, I. Černušák, and P. Zahradník, Int. J. of Quant. Chem. 107, 2133 (2007).

[44] S. Pabst and R. Santra, Phys. Rev. A 81, 065401 (2010). 